\title{
Edge Detection through Fuzzy System using Type I Format
}

\author{
Gurjot Kaur \\ M.Tech Student \\ CSE/IT Deprtment \\ BBSBEC Fatehgarh Sahib
}

\author{
Ishpreet Singh Virk \\ Assistant Professor \\ CSE/IT Deprtment \\ BBSBEC Fatehgarh Sahib
}

\begin{abstract}
This paper proposes the implementation of a very simple but efficient fuzzy logic based algorithm to detect the edges of an image with desired level of threshold values. The approach begins by scanning the images using floating $2 \times 2$ pixel window. FIS is designed with 4 inputs, whose output is further used in proposed algorithm to reduce the noise in the edges produced by the FIS. This algorithm is compared with six standard algorithms and one more system is also proposed which gives the direct comparison of these six algorithms visually.
\end{abstract}

\section{Keywords}

Edge detection, Fuzzy logic, Fuzzy inference system

\section{INTRODUCTION}

Edge detection is a vital task in image processing. It's a main tool in image segmentation, pattern recognition and scene analysis. An edge detector is essentially a high pass filter that may be applied to extract the sting points in a picture. An edge in a picture may be a contour across that the brightness of the image changes suddenly. In image processing, an edge is commonly taken as one class of singularities. In a very function, singularities are characterized simply as discontinuities where the gradient approaches infinity. However, image data is therefore, thus edges in an image usually are outlined as the local maxima of the gradient. Edge widely exists between objects and backgrounds, between objects and primitives. The edge of an object is mirrored within the separation of the gray. Therefore, the overall technique of edge detection is to check the changes of one image pixel in a gray area, which uses the variation of the first-order or second-order edge neighboring to observe the edge [1]. This methodology is employed to refer as local operator edge detection technique. Edge detection is especially the detection, measurement, and location of the changes in image gray.

\section{PRESENT WORK}

In image processing and computer vision, edge detection treats the localization of significant variations of a gray level image and the identification of the physical and geometrical properties of objects of the scene. Edge detection is a difficult issue. Many difficulties come from the complex contents like noise, varying contrast in an image, orientation sensitivity.

Traditional edge detection techniques, such as Robert operator, Sobel operator, Prewitt are widely used. All these techniques are studied and compared with the proposed method using fuzzy inference rule based approach. It is found that most of the existing techniques are either very sensitive to noise and do not give satisfactory results in low contrast areas.

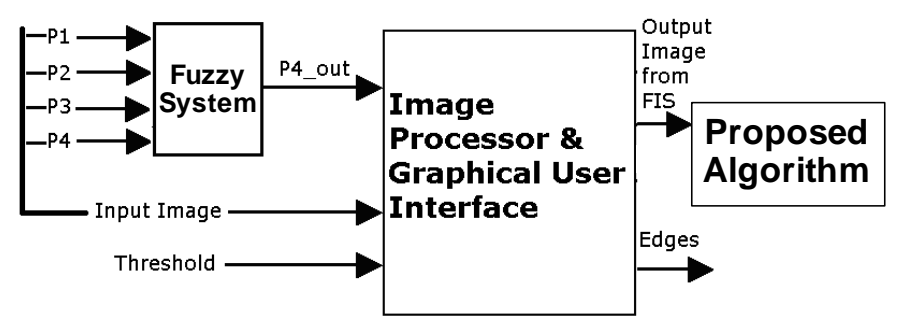

Fig. 1: Block Diagram of Edge Detection through Fuzzy System

Edge detection using fuzzy logic provides an alternative approach to detect edges. A fuzzy theory based Edge Detector avoids these problems and is a better method for edge information detection and noise filtering than the traditional methods.

As Experimental approach is characterized by much greater control over the research environment and in this case some variables are manipulated to observe their effect on other variables. This research is based on experimental research because in this research work we have taken four inputs which are evaluated by sixteen rules of FIS, which give results as edge, black or white and this result is further used in derivative algorithm proposed in this research. Which is finally provided the results. We can present the complete process of research in the form of Flow-Chart. 


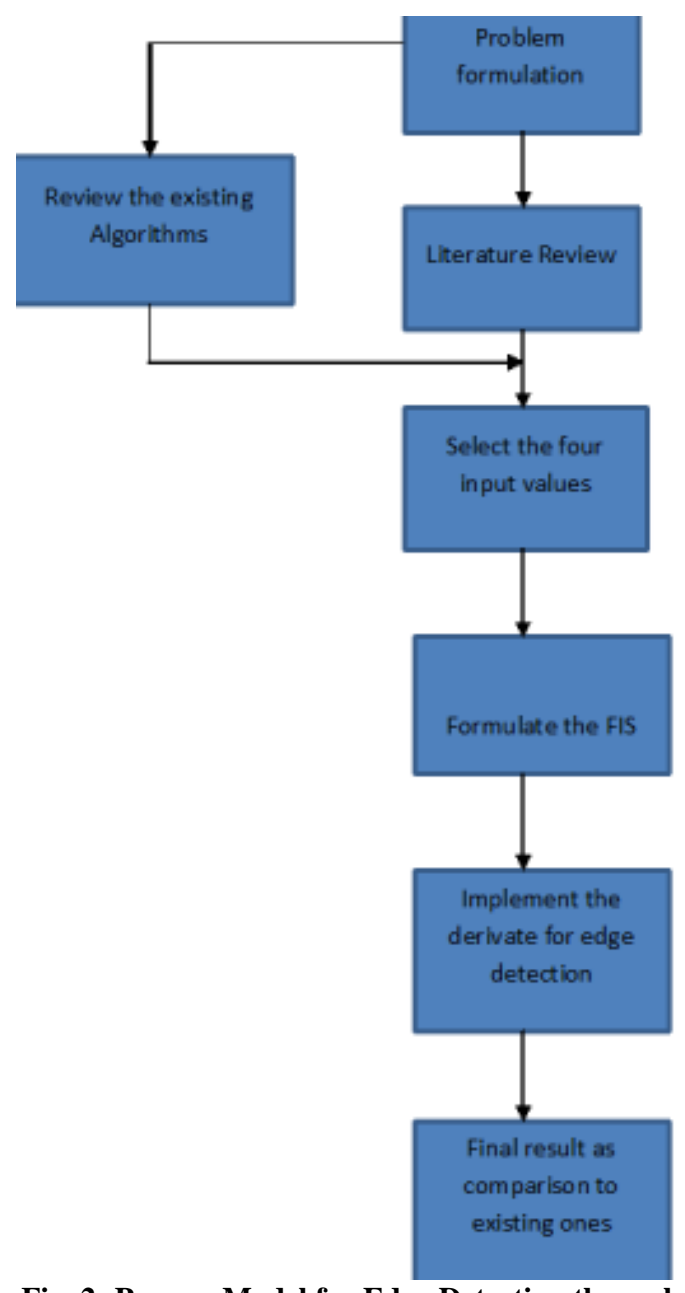

Fig. 2: Process Model for Edge Detection through Fuzzy

\section{IMPLEMENTATION}

This fuzzy rule based edge detection system is developed by designing a Fuzzy Inference System (FIS) of Mandeni type using MATLAB 2008. A smallest possible $2 \mathrm{X} 2$ window is used as a scanning mask as shown in figure below: -

\begin{tabular}{|l|l|}
\hline $\mathrm{P} 1$ & $\mathrm{P} 2$ \\
$\mathrm{Y}(\mathrm{I}, \mathrm{j})$ & $\mathrm{Y}(\mathrm{i}, \mathrm{j}+1)$ \\
\hline $\mathrm{P} 3$ & $\mathrm{P} 4$ \\
$\mathrm{Y}(\mathrm{i}+1, \mathrm{j})$ & $\mathrm{Y}(\mathrm{i}+1, \mathrm{j}+1)$ \\
\hline
\end{tabular}

Fig. 3: Pixel representation for Edge Detection

Mask slides over the full image pixel by pixel, 1st horizontally in upmost horizontal line then once reaching at the end of line, it increments to ascertain succeeding vertical location and it continues until the full image is scanned. The FIS built has four inputs, every input representing a pixel for 2X2 mask, and one output that represents pixel into consideration. The rule editor consists of sixteen fuzzy rules for considering the weights of like P1, P2, P3 and P4 with P4_out for instance if P1, P2, P3 and P4are black then the P4_out is black.

Fuzzy logic is conceptually simple to know and is versatile. The mathematical ideas behind fuzzy reasoning are very straightforward. fuzzy logic is tolerant of general information and fuzzy logic will model nonlinear functions of arbitrary complexity. fuzzy logic relies on natural language i.e. itl's designed atop the structures of qualitative description utilized in everyday language, fuzzy logic is simple to use[8]. In general, the fuzzy logic provides an inference structure that permits appropriate human reasoning capabilities.

On the contrast, in classical set theory i.e. traditional binary set theory, the membership of components in a very set is assessed in binary terms consistent with a bivalent condition i.e. follows 2 valued logic, either true or false - a component either belongs or doesn't belong to the set. It describes crisp i.e. precise events, events that either do or donl't occur. consider ring an example that if ' $a$ ' is member or component of the set ' $\mathrm{A}$ ' then solely 2 possible relationships will exists between ' $a$ ' and ' $A$ ', either ' $a$ ' belongs to ' $A$ ' or ' $a$ ' doesn't belongs to ' $A$ '.

The theory of fuzzy logic relies upon the notion of relative graded membership and then are the functions of mutation and cognitive processes. The utility of fuzzy sets lies in their ability to model unsure or ambiguous data therefore usually encountered in real world. The Fuzzification and Defuzzification steps are owing to the actual fact that we tend to don't possess hardware.

In this paper, the designed fuzzy inference system is given four inputs i.e. P1, P2, P3, P4 and one output i.e. P4_out. The four inputs are the four are values of the $2 \mathrm{X} 2$ windows mask used. The trapezodial membership functions are used each for the inputs and also the output. 2 fuzzy sets i.e. Black and White are used for the input and 3 fuzzy sets i.e. Black, White and Edge are used for the output.

\section{Fuzzy Rules:}

These rules are read as:

Rule 1 : If $\mathrm{P} 1$ is black, $\mathrm{P} 2$ is black, $\mathrm{P} 3$ is black and $\mathrm{P} 4$ is black then output is black.

Rule 2 : If $\mathrm{P} 1$ is white, $\mathrm{P} 2$ is white, $\mathrm{P} 3$ is white and $\mathrm{P} 4$ is white then output is White.

Rule 3 : If $\mathrm{P} 1$ is white, $\mathrm{P} 2$ is white, $\mathrm{P} 3$ is white and $\mathrm{P} 4$ is black then output is Edge.

Rule 4 : If P1 is black, $\mathrm{P} 2$ is black, $\mathrm{P} 3$ is black and $\mathrm{P} 4$ is white then output is Edge.

Rule 5 : If $\mathrm{P} 1$ is black, $\mathrm{P} 2$ is white, $\mathrm{P} 3$ is Black and $\mathrm{P} 4$ is White then output is Edge.

Rule 6 : If $\mathrm{P} 1$ is white, $\mathrm{P} 2$ is black, $\mathrm{P} 3$ is white, $\mathrm{P} 4$ is Black then output is Edge.

Rule 7 : If $\mathrm{P} 1$ is white, $\mathrm{P} 2$ is white, $\mathrm{P} 3$ is white, $\mathrm{P} 4$ is black then output is Edge.

Rule 8 : If $\mathrm{P} 1$ is black, $\mathrm{P} 2$ is Black, $\mathrm{P} 3$ is white, $\mathrm{P} 4$ is white then output is Edge.

Rule 9 : If $\mathrm{P} 1$ is black, $\mathrm{P} 2$ is white, $\mathrm{P} 3$ is white, $\mathrm{P} 4$ is black then output is Edge.

Rule 10 : If $\mathrm{P} 1$ is white, $\mathrm{P} 2$ is black, $\mathrm{P} 3$ is black, $\mathrm{P} 4$ is white then output is Edge. 
Rule 11 : If $\mathrm{P} 1$ is white, $\mathrm{P} 2$ is Black, $\mathrm{P} 3$ is Black, $\mathrm{P} 4$ is Black then output is Black.

Rule 12 : If $\mathrm{P} 1$ is black, $\mathrm{P} 2$ is white, $\mathrm{P} 3$ is black, $\mathrm{P} 4$ is Black then output is Edge.

Rule 13 : If $\mathrm{P} 1$ is Black, $\mathrm{P} 2$ is Black, $\mathrm{P} 3$ is white, $\mathrm{P} 4$ is Black then output is Edge.

Rule 14 : If $\mathrm{P} 1$ is black, $\mathrm{P} 2$ is white, $\mathrm{P} 3$ is white, $\mathrm{P} 4$ is white then output is White.

Rule 15 : If $\mathrm{P} 1$ is white, $\mathrm{P} 2$ is black, $\mathrm{P} 3$ is white, $\mathrm{P} 4$ is white then output is Edge.

Rule 16 : If $\mathrm{P} 1$ is white, $\mathrm{P} 2$ is white, $\mathrm{P} 3$ is black, $\mathrm{P} 4$ is white then output is Edge[12].

The Stepwise procedure is listed below:

Step I: Select the algorithm Sobel, Canny, Prewitt, Zero, Laplacian and Roberts.

Step II : Select the threshold value to some desired level

Step III: Press the OK button

Step IV: Visualize the original image and the output of selected algorithm to which comparison is to be done.

Step V: Wait for the program Status Bar to reach to its final value and compare the output obtained from our program to that of the selected algorithm and make some final conclusion.

\section{RESULT AND DISCUSSIONS}

The algorithm for fuzzy edge detection method is simulated using MATLAB on different images. The resulting images are generated by FIS and proposed algorithm. The system gives the performance based on the threshold values selected by the user. The system is tested with different images, and it was observed that the outputs of this algorithm provide distinct marked edges and thus have better visual appearance. It can be observed that the output that has been generated by the fuzzy method has find the edges of the image more distinctly as compared to the ones that have been found out by the other six edge detection algorithms. Thus the Fuzzy rule based System provides edge detection and has an exhaustive set of fuzzy conditions which helps to extract the edges with a very high efficiency. This system also gives the visual comparison of six of the algorithms defined above separately.

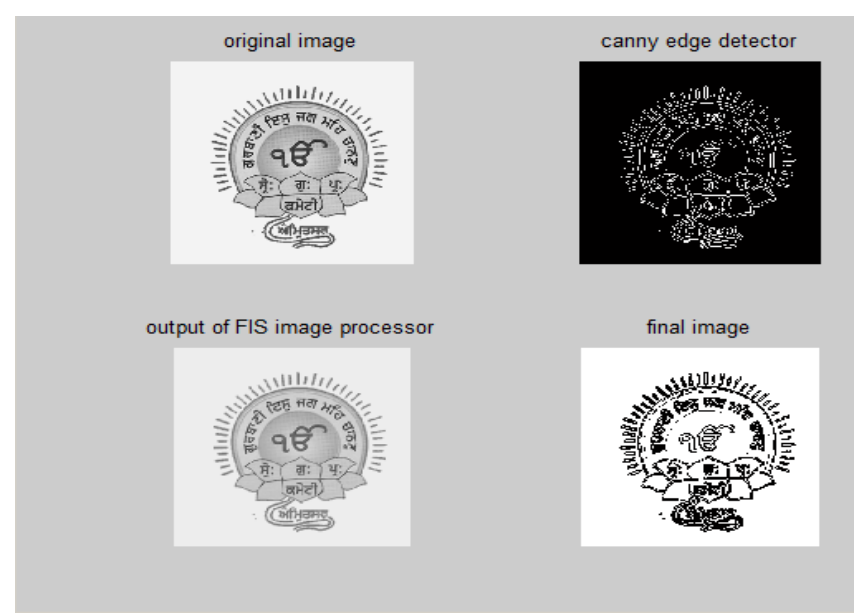

Fig. 4: Result of Proposed Algorithm with comparison to Canny Edge Detector

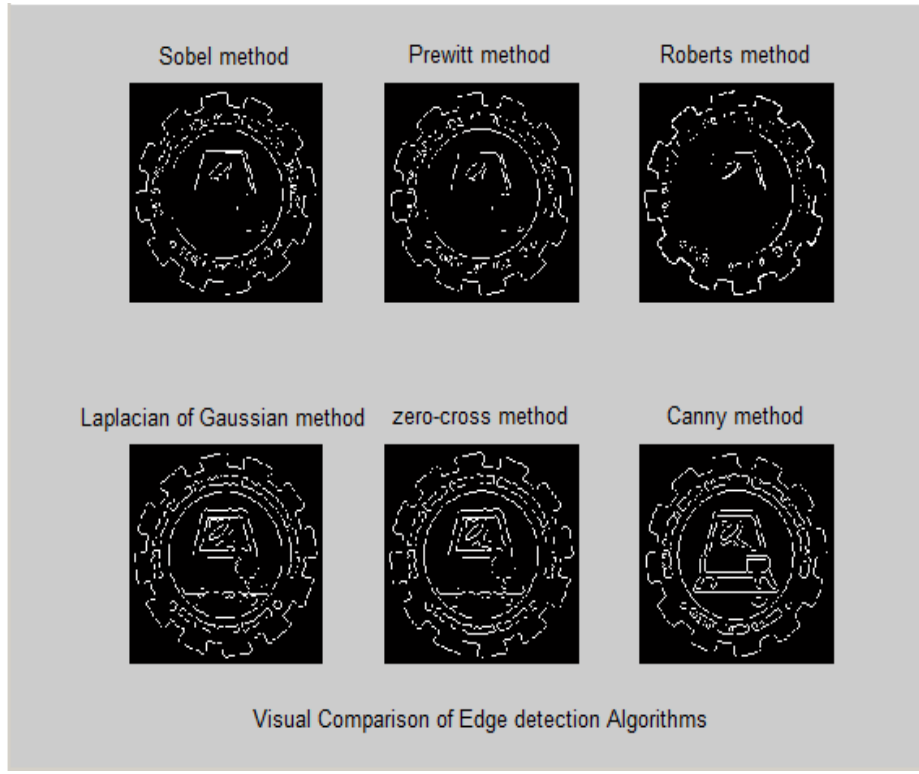

Fig. 5: Visual comparision of six Standard Algorithms like sobel, prewitt, Roberts, LoG, Zero-Cross and Canny

\section{CONCLUSION}

In this paper, edge detection through fuzzy system using type 1 format has been presented. It gives the option to choose the threshold value with the help of slider so thickness can be decided as per requirement. With the help of proposed algorithm noise is reduced to provide the better edges of the image but there is scope of further research remaining.

\section{REFERENCES}

[1] Pooja Sharma,Gurpreet Singh, Amandeep Kaur, "Different Techniques Of Edge Detection In Digital Image Processing", International Journal of Engineering research and Applications, Vol. 3,Issue 3, pp.458461,2013

[2] Li Yinghua , Liu Bingqi and Zhou Bin [2005], "The Application Of Image Edge Detection by using Fuzzy Technique", Electronic Imaging and Multimedia Technology IV, Proceeding of SPIE Vol. 5637, pp.398405 .

[3] Yasar Becerikli, Tayfun M. Karan [2005], "A New Fuzzy Approach for Edge Detection",proceeding of International Conference on Computational Intelligence and Bio Inspired Systems, LNCS, vol.3512, pp.675-709.

[4] Cristiano Jacques Miosso and Adolfo Bauchspiess [2001], "Fuzzy Inference System Applied to edge detection in Digital Images", Proceeding of the V Brazilian Conference on Neural Networks, pp. 481-486.

[5] Todd Law, Hidenori Itoh and Hirohisa Seki ,Member [1996], "Image Filtering, Edge Detection, and Edge Tracing Using Fuzzy Reasoning", IEEE Transactions on Pattern Analysis and Machine Intelligence, Vol. 18(5), pp. 481-491.

[6] Jinbo Wu, Zhouping Yin and Youlun Xiong [200], "The Fast Multilevel Fuzzy Edge Detection of Blurry Images",IEEE letters on Signal Processing,pp.344-347.

[7] M.Hanmandlu, Rohan Raj Kalra, Vamsi Krishna Madasu and S.Vasikarla[2006], "Area Based Novel Approach 
For Fuzzy Edge Detection”, TENCON 2006:2006 IEEE Region 10 Conference, pp. 1-4.

[8] Vishal Paika and Pankaj Bhambri [2013], "Edge Detection-Fuzzy Inference System",International journal of management \& Information Technology Vol.4, no.1 pp. 148-155.

[9] Yasar Becerikli, Tayfun M. Karan and Ali Okatan [2009], "A New Fuzzy Edge Detector for noisy images using modified WFM Filter", International Journal of Innovative Computing,

[10] Fabr izio Russo [1998], "Edge Detection in Noisy Images Using Fuzzy Reasoning", IEEE Transaction on Instrumentation and Measurement, Vol. 47(5),pp.11021105.

[11] Abdallah A. Alshennawy, and Ayman A. Aly [2009], "An Edge Detection and Filtering Mechanism of Two Dimensional Digital Objects Based on Fuzzy Inference",
Information and Control (ICIC) Vol.5(6), pp. 1725-1733. Proceeding of World Academy of Science, Engineering and Technology, pp.247-251.

[12] Abhradita Deepak borkar and mithilesh atulkar [2013], "Fuzzy Inference System for Image Processing" International journal of advanced research in computer engineering \& technology, vol 2, Issue 3, pp. 1007-1010.

\section{AUTHOR'S PROFILE}

Gurjot Kaur is a M.Tech Student in Department of computer science and Engineering in the BBSB Engineering College Fatehgarh Sahib, Punjab Technical University Jalandhar, Punjab. She obtained her M.Tech (E-Security) From PTU, Jalandhar and B.Tech (IT) From PTU Jalandhar.

Ishpreet Singh is working as an assistant professor in the department of computer science and Engineering and Information Technology in Baba Banda Singh Bahadur Engineering College Fatehgarh Sahib, Punjab. 